\title{
A fixed recourse integer programming approach towards a scheduling problem with random data: A case study
}

\author{
JW Joubert* DG Conradie ${ }^{\dagger}$ \\ Received: 4 October 2004; Revised: 17 January 2005; Accepted: 24 January 2005
}

\begin{abstract}
Regardless of the success that linear programming and integer linear programming has had in applications in engineering, business and economics, one has to challenge the assumed reality that these optimization models represent. In this paper the certainty assumptions of an integer linear program application is challenged in an attempt to improve the solution robustness in an uncertain environment. The authors resort to a two-stage, fixed recourse program to introduce random variables with a uniform distribution instead of deterministic expected values in a workforce sizing and scheduling problem. Although the solution to the problem comprises a significantly larger fulltime staff complement than that determined via the problem without the introduction of random variables, the expected workforce requirements preempt and consider the costly expense of casual workers.
\end{abstract}

Key words: stochastic programming, fixed recourse, optimization, integer programming, workforce sizing, scheduling.

\section{Introduction}

The formulation of scheduling problems involves the assignment of scarce resources to complete a number of competing tasks so as to attain a level of performance acceptable to decision-makers. The ground services function at a higher education institution typically oversees the overall tidiness of the university's campuses, and is usually contractually outsourced to a private company. In an attempt to assist the contractor to ensure a favourable outcome when such a contract was reviewed at the University of Pretoria and considered for renewal at the end of 2004, the authors embarked on an optimization procedure in a previous paper [6]. In this previous paper the authors attempted to find the optimal

\footnotetext{
${ }^{*}$ Corresponding author: Department of Industrial Engineering, University of Pretoria, Pretoria, 0002, South Africa, email: johan.joubert@up.ac.za

${ }^{\dagger}$ Department of Industrial Engineering, University of Pretoria, Pretoria, 0002, South Africa
} 
number of full-time workers that were required during weekdays and weekends, respectively, so that all the work stipulated by the service level agreement would be completed. The objective of the optimization model was to find the least-cost full-time staff complement. Although the model produced a detailed and predictive schedule that was used to provide visibility of the day-to-day schedule, the strategic intent of the decision model was the capacity check that indicated to management what staff complement to employ. The purpose of the scheduling endeavour is based on the taxonomy provided by Aytug et al. (2005). As opposed to the Service Case Scheduling Problem (SCSP), as proposed by Simmons et al. (2004), where a service provider manages a number of customer cases, the project described above was concerned with the scheduling of the actual service workers, and providing management with an aggregated capacity check to determine and predict the staff complement.

The initiative described in this paper was fueled by an attempt to expose undergraduate students to a more realistic problem-solving environment by addressing the curriculation of the undergraduate modules in Operations Research in the Department of Industrial and Systems Engineering at the University of Pretoria. The encouraging results of the recurriculation of the modules, and the introduction of a comprehensive semester project, were published by Joubert and Steyn (2003).

This paper is structured as follows. The original integer linear program is stated in Section 2. The deterministic time required for performing tasks is challenged in Section 4, by introducing random time requirements through a two-stage fixed recourse program. A similar approach is followed in Section 5, where a random time-based percentage is introduced to determine tool requirements. The way in which the random distributions are approximated by discrete distributions, as well as which random variables were introduced, are discussed in Section 6. The results and a few decision implications conclude this paper in Section 7, where suggestions for future research and proposed model refinements are also given.

\section{The original problem}

In the original problem, Conradie and Joubert (2004) defined the decision variables as

$$
x_{i j k m} \triangleq \begin{cases}1 \quad \text { if worker } k \text { does job } i \text { in period } j \text { of season } m, \text { where } i=\{1,2, \ldots, 5\} \\ j=\{1,2, \ldots, 12\}, k=\{1,2, \ldots, 50\}, \text { and } m=\{1,2\} \\ 0 \quad \text { otherwise }\end{cases}
$$

and

$$
y_{i k m} \triangleq \begin{cases}1 \quad \text { if worker } k \text { does job } i \text { in season } m, \text { where } i=\{1,2, \ldots, 5\} \\ k=\{1,2, \ldots, 50\}, \text { and } m=\{1,2\} \\ 0 \quad \text { otherwise }\end{cases}
$$


Other model parameters include

$p_{i j m} \triangleq$ the number of workers required to perform task $i$ in period $j$ of season $m$, where $i=\{1,2, \ldots, 5\}, j=\{1,2, \ldots, 12\}$ and $m=\{1,2\}$,

$w_{j m} \triangleq$ the number of workers required during weekdays (time period $j$ ) in season $m$, where $j=\{1,2, \ldots, 10\}$ and $m=\{1,2\}$,

$q \triangleq$ the maximum number of workers required during weekdays,

$s_{j m} \triangleq$ the number of workers required on Saturdays (time period $j$ ) in season $m$, where $j=\{1,2, \ldots, 10\}$ and $m=\{1,2\}$,

$r \triangleq$ the maximum number of workers required on Saturdays,

$t_{i m} \triangleq$ the number of person-periods required for task $i$ in season $m$, where $i=\{1,2, \ldots, 5\}$ and $m=\{1,2\}$,

$n_{\alpha} \triangleq$ the number of tools of type $\alpha$ available, where $\alpha=\{1,2, \ldots, 8\}$,

$b_{i m \alpha} \triangleq$ the time-based percentage of a period that task $i$ requires tool $\alpha$ in season $m$, where $i=\{1,2, \ldots, 5\}, m=\{1,2\}$ and $\alpha=\{1,2, \ldots, 8\}$.

The authors challenge a perfectly balanced schedule and formulated the workforce sizing and scheduling problem as

$$
\min z=5 c_{1} q+c_{2} r,
$$

subject to

$$
\begin{array}{rlrl}
\sum_{i=1}^{5} p_{i j m} \leq w_{j m}, & j \in\{1,2, \ldots, 10\}, m \in\{1,2\} \\
w_{j m} \leq q, & j \in\{1,2, \ldots, 10\}, m \in\{1,2\} \\
\sum_{i=1}^{5} p_{i j m} \leq s_{j m}, & j \in\{11,12\}, m \in\{1,2\} \\
s_{j m} \leq r, & j \in\{11,12\}, m \in\{1,2\} \\
\sum_{j=1}^{12} p_{i j m} \geq t_{i m}, & i \in\{1,2, \ldots, 5\}, m \in\{1,2\} \\
\sum_{i=1}^{5} b_{i m \alpha} p_{i j m} \leq n_{\alpha}, & j \in\{1,2, \ldots, 12\}, m \in\{1,2\}, \alpha \in\{1,2, \ldots, 8\} \\
\sum_{k=1}^{50} x_{i j k m} & =p_{i j m}, & & i \in\{1,2, \ldots, 5\}, j \in\{1,2, \ldots, 12\}, m \in\{1,2\} \\
\sum_{h=1}^{3} x_{i, h+j-1, k, m} \leq 2, & & i \in\{1,2, \ldots, 5\}, j \in\{1,2, \ldots, 10\}, \\
\sum_{i=1}^{5} x_{i j k m} \leq 1, & & j \in\{1,2, \ldots, 50\}, m \in\{1,2\}
\end{array}
$$




$$
\begin{array}{cl}
\sum_{j=1}^{12} x_{i j k m} \leq M y_{i k m}, & i \in\{1,2, \ldots, 5\}, k \in\{1,2, \ldots, 50\}, m \in\{1,2\} \\
\sum_{i=1}^{5} y_{i k m} \geq 3, & k \in\{1,2, \ldots, 50\}, m \in\{1,2\} \\
b_{i m \alpha} \geq 0, & i \in\{1,2, \ldots, 5\}, m \in\{1,2\}, \alpha \in\{1,2, \ldots, 8\} \\
x_{i j k m}, y_{i k m} \in\{0,1\}, & i \in\{1,2, \ldots, 5\}, j \in\{1,2, \ldots, 12\}, \\
& k \in\{1,2, \ldots, 12\}, m \in\{1,2\} \\
p_{i j m}, t_{i m}, w_{j m}, s_{j m}, n_{\alpha} \geq 0 \text { and integer, } \quad & i \in\{1,2, \ldots, 5\}, j \in\{1,2, \ldots, 12\}, \\
& m \in\{1,2\}, \alpha \in\{1,2, \ldots, 8\},
\end{array}
$$

where $M$ is a large number. The objective function represented in (1) minimises the total number of full-time workers employed, where $c_{1}$ and $c_{2}$ denote the daily wage for workers employed on weekdays and on Saturdays, respectively, with constraints (2) through (5) determining the number of workers employed on the various days of the week. The minimum time allocation for each task is enforced by (6), with the assumption that one person working one period fulfils one person-period worth of work. The tools required for each task are stipulated by (7). Workers and tasks are matched in (8), while task repetition is addressed in (9). A worker is only human according to (10), while (11) and (12) attempt to assign workers in a multi-skilled fashion [11].

The solution to the original problem saw 20 full-time workers employed during the week $(q=20)$, and no full-time workers employed over weekends $(r=0)$. The original problem did not take casual workers into account, as all tasks were assumed to be executed by fulltime employees as per schedule. Aytug et al. (2005) confirm in their review that schedules are often not adhered to. The private contractor required the services of casual workers over weekends to fulfil service obligations that could not be achieved during weekdays by full-time employees. The service backlog is attributed to absenteeism, irregular weather patterns, and events with assignable causes.

\section{A random environment}

The results of the original problem proved somewhat unreliable in that the ground services company had to rely on casual workers over weekends to catch up on underperformance, necessitating some investigation into the model's underlying assumptions. The decision to employ casual workers was a purely reactive decision. It was apparent that a situation in which the execution is reliant on human action, cannot and should not assume process times and parameters to be known and certain. In their comprehensive review article that focussed more on scheduling within the manufacturing realm, Aytug et al. (2005) distinguish between research papers in scheduling as belonging to either deterministic or stochastic approaches, and develop a taxonomy for classifying scheduling problem approaches. Their paper's implication is not limited to the manufacturing environment, and valuable insight is to be gained from the taxonomy irrespective of the area of application. As opposed to a pure stochastic model that assumes all variables to be stochastic 
in nature, and which primarily focusses on control policies, the authors of this paper extended the original deterministic approach of Conradie and Joubert (2004) to a situation containing some form of uncertainty. We support the view that a system that works in a deterministic environment can be engineered to work under at least certain stochastic conditions.

Decision makers often prefer a schedule with suboptimal expected performance if such a schedule limits the performance variability, over a schedule with an optimal expected performance, but with high variability $[7,8]$. A predictive schedule is generated, and some reactive measures are required only once a specific random parameter value has realized. In an uncertain environment, each possible realization will result in some reactive measure. The cost of reacting to the possible realizations are then minimized.

Stochastic integer linear programs are integer linear programs in which some of the parameters and problem data are considered uncertain. Recourse programs are those in which some decisions or recourse can be taken after the extent of the uncertainty is disclosed [5]. The private company under consideration wishes to make a decision regarding its full-time staff complement. A predictive schedule is generated that does not only provide visibility of the detail task scheduling, but also indicate the aggregated capacity required to fulfil the contractual obligations under expected conditions. However, as reality unfolds with absent staff and unforseen weather implications, they are required to take reactive action to ensure that they adhere to their contractually established performance requirements. Casual workers are used to fulfil the additional labour requirements, but are more costly than full-time employees, as the tax and financial benefit burden becomes the responsibility of the casual worker. Furthermore, casual workers' employment stability and security are compensated for by means of a financial wage premium. The employment of casual workers therefore become the recourse taken to ensure that adequate service levels are attained. Bard et al. (2001) also include the issue of addressing schedule disruptions within the scope of their study. Over and above the inclusion of temporary workers, Xinhui and Bard (2005) have also distinguished between full-time and part-time employees, a situation that falls outside the scope of this project.

Although Bard (2004) and Bechtold and Brusco (1994) also determine the optimal staff complement, a multi-skilled workforce is assumed. Whenever a worker is assigned a task rated at a lower skill level than the worker's ability (i.e., downgrading occurs), a penalty is incurred. Such a distinction between skill levels is omitted from this paper.

\section{Introducing random task requirements}

In two-stage recourse models one explicitly distinguishes between decision variables based on whether they are implemented before or after an outcome value of a random variable is observed. The objective of the modelling endeavour is to assist management of the outsourced company to decide on the first-stage variables, $x_{i j k m}$ (i.e., how many workers to employ during the various task periods). This is achieved by determining the predictive schedule, and has to be made prior to the realization of random elements that influence the model. Second-stage decision variables are regarded as reactive and are associated with the additional $a d$-hoc casual workers, or the overtime of employed workers, to compensate 
for loss of maintenance activity.

The authors challenge the appropriateness of constraint (6) by not using the expected value of the number of periods required for task $i$ in season $m$, denoted by $t_{i m}$, and in doing so introduce a random variation on the value, such that

$$
t_{i m}=\tilde{\zeta}_{i m}, \quad i \in\{1,2, \ldots, 5\}, m \in\{1,2\},
$$

where the random variables $\tilde{\zeta}_{i m}$ are modelled using uniform distributions. The introduction of the random variables into $(6)$ sees the introduction of a recourse variable $\omega_{i m}\left(\tilde{\zeta}_{i m}\right)$, which simply measures the number of casual workers required to perform task $i$ in season $m$. Since the shortage of workers depends on a realization of $\tilde{\zeta}_{i m}$, so does the corresponding recourse variable and since we are only interested in tracking the positive number of casual workers required, we replace the deterministic constraints introduced in (6) by

$$
\omega_{i m}\left(\tilde{\zeta}_{i m}\right)=\omega_{i m}^{+}\left(\tilde{\zeta}_{i m}\right)-\omega_{i m}^{-}\left(\tilde{\zeta}_{i m}\right)
$$

such that

$$
\sum_{j=1}^{12} p_{i j m}+\omega_{i m}^{+}\left(\tilde{\zeta}_{i m}\right)-\omega_{i m}^{-}\left(\tilde{\zeta}_{i m}\right)=\tilde{\zeta}_{i m}, \quad i \in\{1,2, \ldots, 5\}, m \in\{1,2\}
$$

with the additional requirement that

$$
\omega_{i m}^{+}\left(\tilde{\zeta}_{i m}\right), \omega_{i m}^{-}\left(\tilde{\zeta}_{i m}\right) \geq 0, \quad i \in\{1,2, \ldots, 5\}, m \in\{1,2\} .
$$

Instead of the ten single random variables $\tilde{\zeta}_{11}, \tilde{\zeta}_{12}, \tilde{\zeta}_{21}, \ldots, \tilde{\zeta}_{52}$, it seems convenient to use a random vector $\tilde{\xi} \in\left(\tilde{\zeta}_{11}, \tilde{\zeta}_{12}, \tilde{\zeta}_{21}, \ldots, \tilde{\zeta}_{52}\right)^{T}$. We assume that casual workers on Saturdays are paid 1.5 times the amount that employed workers are paid for the same period, and the objective function of the resulting resource program then becomes

$$
\min z=5 c_{1} q+c_{2} r+\sum_{i=1}^{5} \sum_{m=1}^{2} E_{\tilde{\xi}}\left[1.5 c_{2} \omega_{i m}^{+}(\tilde{\xi})\right]
$$

where $E_{\tilde{\xi}}$ represents the expected value with respect to the distribution of $\tilde{\xi}$. If $\xi^{\gamma}$ represents the $\gamma^{\text {th }}$ realization of $\tilde{\xi}$, which has a finite discrete distribution $\left\{\left(\xi^{\gamma}, p_{\gamma}\right), \gamma=1, \ldots, R\right\}$ with $\left(p_{\gamma}>0\right.$ for all $\left.\gamma\right)$, we may establish an ordinary mixed integer program with a dual composition structure whose objective function is

$$
\min z=5 c_{1} q+c_{2} r+\sum_{i=1}^{5} \sum_{m=1}^{2} \sum_{\gamma=1}^{R} p_{\gamma}\left[1.5 c_{2}\left(\omega_{i m}^{+}\left(\xi^{\gamma}\right)\right)\right] .
$$

The constraints related to the random task requirements then become

$$
\begin{gathered}
\sum_{j=1}^{12} p_{i j m}+\omega_{i m}^{+}\left(\xi^{\gamma}\right)-\omega_{i m}^{-}\left(\xi^{\gamma}\right)=\xi^{\gamma}, \quad i \in\{1,2, \ldots, 5\}, m \in\{1,2\}, \gamma \in\{1,2, \ldots, R\} \\
\omega_{i m}^{+}\left(\xi^{\gamma}\right), \omega_{i m}^{-}\left(\xi^{\gamma}\right) \geq 0, \quad \gamma \in\{1,2, \ldots, R\}
\end{gathered}
$$


with all the other constraints remaining as per the original formulation.

\section{$5 \quad$ Introducing random time-based percentages}

Although slightly more complex from a computational point of view, the validity of the assumption that the time-based percentage that tool type $\alpha$ will be used in task $i$ of season $m$, namely $b_{i m \alpha}$, as expressed in the original constraint (7) is also challenged in this section.

A random variable $\tilde{\beta}_{i m \alpha}$ is introduced to replace the deterministic value $b_{i m \alpha}$, and is again modelled using uniform distributions. We introduce a recourse variable $z_{j m \alpha}\left(\tilde{\beta}_{i m \alpha}\right)$, which determines the number of tools of type $\alpha$ that one would outsource (or rent) in period $j$ of season $m$. As in the case of the time requirements, the additional tools required are dependent on a realization of $\tilde{\beta}_{i m \alpha}$, and so are the corresponding recourse variables. As opposed to being interested in the positive occurrences of $\omega_{i m}\left(\tilde{\zeta}_{i m}\right)$ in $(18)$, we are interested in the negative occurrences of $z_{j m \alpha}\left(\tilde{\beta}_{i m \alpha}\right)$. We thus replace $(7)$ by

$$
z_{j m \alpha}\left(\tilde{\beta}_{i m \alpha}\right)=z_{j m \alpha}^{+}\left(\tilde{\beta}_{i m \alpha}\right)-z_{j m \alpha}^{-}\left(\tilde{\beta}_{i m \alpha}\right),
$$

such that

$$
\sum_{i=1}^{5} \beta_{i m \alpha} p_{i j m}+z_{j m \alpha}^{+}\left(\tilde{\beta}_{i m \alpha}\right)-z_{j m \alpha}^{-}\left(\tilde{\beta}_{i m \alpha}\right)=n_{\alpha}
$$

for all $j \in\{1,2, \ldots, 12\}, m \in\{1,2\}, \alpha \in\{1,2, \ldots, 8\}$. We have to introduce the additional constraints

$$
z_{\text {jma }}^{+}, z_{\text {jm } \alpha}^{-} \geq 0 \text { and integer, } \quad j \in\{1,2, \ldots, 12\}, m \in\{1,2\}, \alpha \in\{1,2, \ldots, 8\}
$$

for the recourse variables. It is again convenient to include the random variables $b_{i m \alpha}$ into a random vector, $\tilde{\xi}$, such that $\tilde{\xi} \in\left(\tilde{\zeta}_{11}, \ldots, \tilde{\zeta}_{52}, \tilde{\beta}_{111}, \tilde{\beta}_{112}, \ldots, \tilde{\beta}_{528}\right)^{T}$. The ordinary mixed integer program with dual composition structure is then expressed as

$$
\min z=5 c_{1} q+c_{2} r+\sum_{i=1}^{5} \sum_{j=1}^{12} \sum_{m=1}^{2} \sum_{\alpha=1}^{8} \sum_{\gamma=1}^{R} p_{\gamma}\left[1.5 c_{2}\left(\omega_{i m}^{+}\left(\xi^{\gamma}\right)\right)+c_{3}^{\alpha}\left(z_{j m \alpha}^{-}\left(\xi^{\gamma}\right)\right)\right]
$$

subject to

$$
\begin{array}{rlrl}
\sum_{i=1}^{5} \xi^{\gamma} p_{i j m}+z_{j m \alpha}^{+}\left(\xi^{\gamma}\right)-z_{j m \alpha}^{-}\left(\xi^{\gamma}\right)=n_{\alpha}, & & j \in\{1,2, \ldots, 12\}, m \in\{1,2\}, \\
& \alpha \in\{1,2, \ldots, 8\}, \gamma \in\{1,2, \ldots, R\} \\
z_{j m \alpha}^{+}\left(\xi^{\gamma}\right), z_{j m \alpha}^{-}\left(\xi^{\gamma}\right) \geq 0, & \gamma \in\{1,2, \ldots, R\}
\end{array}
$$


with the cost of renting a tool of type $\alpha$ for one scheduling period being denoted by $c_{3}^{\alpha}$, and the tool constraints expressed in (28) and (29). With the exception of the adapted task requirements, the original constraint formulation remains intact. The range $\gamma \in$ $\{1,2, \ldots, R\}$ is dependent on the discrete distribution of the realizations of $\tilde{\xi}$.

\section{Case study}

We approximate the uniform distributions of the random variables by discrete distributions in such a manner that $R=8$ in $\left\{\left(\xi^{\gamma}, p_{\gamma}\right), \gamma=1, \ldots, R\right\}$ with $\left(p_{\gamma}>0\right.$ for all $\left.\gamma\right)$, as suggested by Kall and Wallace (1994).

For this purpose we generate large samples for $\zeta_{i m}^{\mu}, i \in\{1,2, \ldots, 5\}, m \in\{1,2\}$, and $\mu=\{1,2, \ldots, K\}$, with the sample size $K=1000$ chosen arbitrarily for this paper. We choose equidistant partitions for each distribution and partition each into $r_{i m}$ subintervals. In all the calculations for this paper we have chosen $r_{i m}=R=8$ for all $i \in\{1,2, \ldots, 5\}$, $m \in\{1,2\}$ arbitrarily. We calculate, for each subinterval $I_{i m \gamma}, i=\{1,2, \ldots, 5\}, m=$ $\{1,2\}, \gamma=\left\{1, \ldots, r_{i m}\right\}$, the arithmetic mean $\bar{\zeta}_{i m}^{\gamma}$ of sample values $\zeta_{i m}^{\gamma} \in I_{i m \gamma}$, yielding the estimate for the conditional expectation of $\tilde{\zeta}_{i m}$. For every subinterval we calculate the relative frequency, $p_{i m \gamma}$ for $\zeta_{i m}^{\gamma} \in I_{i m \gamma}$ such that

$$
p_{i m \gamma}=\frac{k_{i m \gamma}}{K}, \quad i \in\{1,2, \ldots, 5\}, m \in\{1,2\}, \gamma \in\left\{1, \ldots, r_{i m}\right\},
$$

where $k_{i m \gamma}$ is the number of sample values $\zeta_{i m}^{\gamma}$ contained in subinterval $I_{i m \gamma}$. These discrete distributions are then used as approximations of the uniform distributions for the random task requirements, as indicated in Table 1 . Here $U(\cdot, \cdot)$ represents a uniform distribution over the stated interval.

\begin{tabular}{lll}
\cline { 1 - 1 }$\tilde{\zeta}_{11} \sim U(120,125)$ & & $\tilde{\zeta}_{32} \sim U(65,80)$ \\
$\tilde{\zeta}_{12} \sim U(40,55)$ & & $\widetilde{\zeta}_{41} \sim U(30,40)$ \\
$\widetilde{\zeta}_{21} \sim U(80,90)$ & & $\widetilde{\zeta}_{42} \sim U(50,60)$ \\
$\tilde{\zeta}_{22} \sim U(105,110)$ & & $\tilde{\zeta}_{51} \sim U(10,20)$ \\
$\tilde{\zeta}_{31} \sim U(40,50)$ & & $\tilde{\zeta}_{52} \sim U(25,35)$ \\
\hline
\end{tabular}

Table 1: Uniform distributions for the random task requirements.

The same procedure is followed to approximate the uniform distributions for the timebased percentages that a task will require a specific type of tool. The difference in approach is that we only consider random variables for limited resources of type $\alpha=\{1\}$, as these are expensive to procure or acquire for a short rental period. The constraints (7) and (28) are thus combined in a manner such that

$$
\begin{aligned}
\sum_{i=1}^{5} \xi^{\gamma} p_{i j m}+z_{j m \alpha}^{+}\left(\xi^{\gamma}\right)-z_{j m \alpha}^{-}\left(\xi^{\gamma}\right)=n_{\alpha}, \quad j \in\{1, \ldots, 12\}, m \in\{1,2\}, \\
\alpha \in\{1\}, \gamma \in\{1, \ldots, 8\}
\end{aligned}
$$




$$
\begin{aligned}
& \text { Improving an ILP application's reliability with stochastic programming } \\
& \sum_{i=1}^{5} b_{i m \alpha} p_{i j m} \leq n_{\alpha}, \quad j \in\{1, \ldots, 12\}, m \in\{1,2\}, \alpha \in\{2, \ldots, 8\}
\end{aligned}
$$

to accommodate random variables in only a limited number of instances. The uniform distributions that are approximated for the time-based percentages are indicated in Table 2.

$$
\begin{aligned}
& \hline \tilde{\beta}_{111} \sim U(0.45,0.55) \\
& \tilde{\beta}_{121} \sim U(0.07,0.10) \\
& \hline
\end{aligned}
$$

Table 2: Uniform distributions for the time-based percentages.

The proposed solution was obtained using a branch-and-bound algorithm and indicates a requirement of $q=31$ full-time weekday workers, and $r=7$ full-time weekend workers, yielding a minimum expected weekly wage bill of R13640. The incumbent solution was obtained by programming the integer linear program (ILP) in $L I N G O^{\mathrm{TM}}$, and interrupting the branch-and-bound solution process after a time limit of six hours. The best solution found after the time limit (incumbent) was taken as the proposed solution. The incumbent solution is thus an upper bound on the solution of the ILP. The objective function value of this solution is in excess of $13.6 \%$ higher than the original $q=20$ full-time weekday workers and $r=0$ full-time weekend workers that yielded theoretical weekly wages of R12 000. It is argued that the solution proposed in this paper is advantageous in a number of ways. Not only does it optimize the expected labour costs by taking the stochastic nature of events into account, thereby addressing expected casual worker requirements that were omitted in the original problem formulation, but the solution also proposes an increased staff complement.

\section{Conclusion}

Although in itself a higher staff complement increases cost and negatively impacts on the myopic objective function, it addresses unemployment issues in South Africa that could assist and benefit the actual implementation of the solution. Quantified solutions provided by Operations Research practitioners (in this case a low wage cost solution) are often disregarded in favor of suboptimal solutions (i.e., higher wage cost alternatives) generated without the use of mathematical optimisation, that can be implemented without labour resistance. Instead of relying heavily on more costly casual labour to address arrears in maintenance performance, the service contractor can employ in excess of ten full-time workers more. Robbins (1996) emphasizes the value of work stability and job security on personal productivity. A larger staff complement thus contributes towards reducing unemployment, and is expected to be adopted more readily by the service contractor.

Investigating the solution found to the problem, it is interesting to observe that the equipment for which random time-based percentages were introduced, did not impact the final solution significantly, challenging the general perception that expensive equipment (or the lack thereof) hampers organizational performance. It is recommended for future research that other equipment requirements be challenged similarly to identify other potential scarce resources. This, however, requires an exorbitant amount of computer processing 
time. Given the solution strategy employed in this paper to obtain an incumbent solution, it is proposed that a metaheuristic optimization algorithm rather be used to find a solution. Although such an endeavour will decrease the computational burden of the algorithm, it may also impact the quality of the current incumbent solution negatively, as it may not converge to the global optimum.

The intent of this research paper was to predict a workforce schedule in an environment where some parameters (in this case the process times and the related equipment requirements) are considered stochastic in nature. In the absence of more accurate data, these parameters were assumed to be uniformly distributed. It would indeed be interesting to investigate the effects of introducing other random distributions, if more data were available to justify a particular choice of random distribution.

As opposed to focussing only on the predictive schedule that provides management with support for capacity decisions, the authors propose that the schedule be used to obtain visibility on day-to-day task, so as to perform micro-management [1]. An example of micromanagement is identifying scarce equipment resources that need to be transported across a distance to be available in geographically dispersed locations in consecutive scheduling periods.

Practitioners in the fields of Industrial Engineering and Operations Research are given an application of stochastic programming in this paper. The authors challenge the notion that marginalizing labour costs often result in better solutions, especially in real applications where human intervention and human reliance are of utmost importance, and where random events hamper the quality and robustness of deterministic solutions.

\section{Acknowledgement}

The authors would like to thank the editor, as well as the two anonymous referees for their valuable inputs and recommendations to improve the quality of this paper.

\section{References}

[1] Aytug H, Lawley MA, McKay K, Mohan S \& Uzsoy R, 2005, Executing production schedules in the face of uncertainties: A review and some future directions, European Journal of Operational Research, 161(1), pp. 86-109.

[2] BARD JF, 2004, Staff scheduling in high volume service facilities with downgrading, IIE Transactions, 36(10), pp. 985-997.

[3] Bard JF, Yu G \& ArgüEllo MF, 2001, Optimizing aircraft routings in response to groundings and delays, IIE Transactions, 33(10), pp. 931-947.

[4] Bechtold SE \& Brusco MJ, 1994, A microcomputer-based heuristic for tour scheduling of a mixed workforce, Computers \& Operations Research, 21(9), pp. 10011009. 
[5] Birge JR \& Louveaux F, 1997, Introduction to stochastic programming, Springer Series in Operations Research, Springer-Verlag, New York (NY).

[6] Conradie DG \& Joubert JW, 2004, Workforce sizing and scheduling for a service contractor using integer programming, South African Journal of Industrial Engineering, 15(2), pp. 133-140.

[7] Daniels RL \& CARRILlo JE, 1997, $\beta$-robust scheduling for single-machine systems with uncertain processing times, IIE Transactions, 29(11), pp. 977-981.

[8] Daniels RL \& Kouvelis P, 1995, Robust scheduling to hedge against processing time uncertainty in single-stage production, Management Science, 41(2), pp. 363376.

[9] Joubert JW \& STEyn AB, 2003, Operationalising operations research, World Transactions on Engineering and Technology Education, 2(1), pp. 91-94.

[10] Kall P \& Wallace SW, 1994, Stochastic programming, John Wiley \& Sons, Chichester.

[11] Robbins AP, 1996, Organizational behaviour, $7^{\text {th }}$ Edition, Prentice-Hall, UpperSaddle River (NJ).

[12] Sen S \& Higle JL, 1999, An introductory tutorial on stochastic linear programming models, Interfaces, 29(2), pp. 33-61.

[13] Simmons JV, Russel GR \& Kraus ME, 2004, Service case scheduling: an exploratory description and recommendations for solution, International Journal of Service Industry Management, 15(4), pp. 385-399.

[14] Xinhui Z \& BARD JF, 2005, Equipment scheduling at mail processing and distribution centers, IIE Transactions, 37(2), pp. 175-187. 
